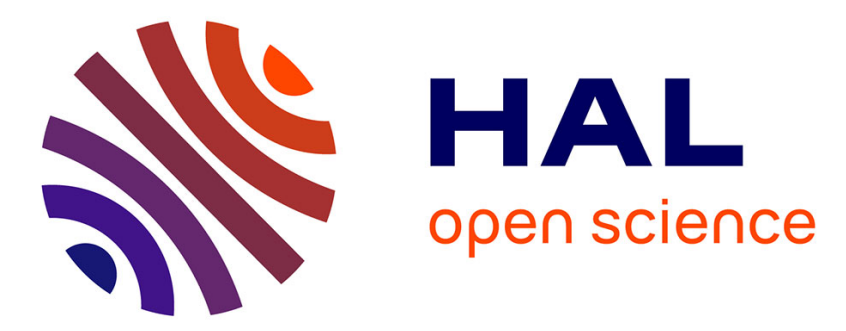

\title{
On correlation functions in random magnets
}

Bernard Derrida, H Hilhorst

\section{To cite this version:}

Bernard Derrida, H Hilhorst. On correlation functions in random magnets. Journal of Physics C: Solid State Physics, 1981, 14 (19), pp.L539-L544. 10.1088/0022-3719/14/19/004 . hal-03285936

\section{HAL Id: hal-03285936 https://hal.science/hal-03285936}

Submitted on 19 Jul 2021

HAL is a multi-disciplinary open access archive for the deposit and dissemination of scientific research documents, whether they are published or not. The documents may come from teaching and research institutions in France or abroad, or from public or private research centers.
L'archive ouverte pluridisciplinaire HAL, est destinée au dépôt et à la diffusion de documents scientifiques de niveau recherche, publiés ou non, émanant des établissements d'enseignement et de recherche français ou étrangers, des laboratoires publics ou privés. 


\title{
LETTER TO THE EDITOR
}

\section{On correlation functions in random magnets}

\author{
Bernard Derrida $\uparrow$ and Henk Hilhorst $\ddagger$ \\ $\dagger$ Service de Physique Théorique, CEN, Saclay, Boite Postale No 2, 91190 Gif-sur- \\ Yvette, France \\ $\$$ Laboratorium voor Technische Natuurkunde, Postbus 5046, 2600 GA Delft, The \\ Netherlands
}

Received 2 March 1981

\begin{abstract}
In random magnets the probability distribution of the correlation function at large distance is not concentrated around its average. To illustrate this idea we study two examples: a random Ising chain and a random cubic chain. The extension of our findings to higher dimension and the connection to Harris' criterion are discussed heuristically. We conclude that in Monte Carlo simulations, due to the combined effects of randomness and finite size effects, one can only measure the most probable value of the correlation functions and not their average.
\end{abstract}

It is well known that, in the study of random magnets like spin glasses (Edwards and Anderson 1975), one has to take into account the probability distribution of the partition function $Z$. In all cases where the interactions are short-range, the distribution $Z$ is sharply peaked around $\exp (\ln Z)$, which generally differs from the average partition function $\bar{Z} \S$. For this reason one has to average the free energy. To our knowledge, this is less justified in the case of long-range interactions like the Sherrington-Kirkpatrick model (1975), because one can no longer use the fact that a large system can be decomposed into weakly interacting subsystems. In all cases, the important quantity is the value of $Z$ around which the probability distribution is peaked; in the thermodynamic limit this peak becomes infinitely sharp and is the only value which can be observed.

In general correlation functions have non-trivial probability distributions even in the thermodynamic limit (see e.g. McCoy and Wu 1968). The purpose of this Letter is to point out that for a correlation function at a large distance $R$, the probability distribution is also sharply concentrated around a value which generally differs from the average. To illustrate this fact, we study two simple examples: an Ising chain with random nearestneighbour interactions and a random cubic chain. In these two cases, we observe that for the correlation function $\left\langle\sigma_{0} \sigma_{R}\right\rangle$ between two spins at distance $R$, the probability distribution is concentrated for large $R$ around $\exp \overline{\left(\ln \left\langle\sigma_{0} \sigma_{R}\right\rangle\right)}$, which differs from $\left\langle\overline{\left.\sigma_{0} \sigma_{R}\right\rangle}\right.$. This remains probably true in most random magnets. It raises the question of whether one must define the correlation length from the average correlation function $\left\langle\overline{\sigma_{0} \sigma_{R}}\right\rangle$ or from its most probable value. We think that both quantities have physical interest and we will discuss their properties in our examples. Finally we discuss heuristically how our conclusions may be extended to higher dimension.

$\S$ Throughout this Letter, the bar indicates an average over the disorder and the brackets mean a thermal average. 
Our first example is an Ising chain

$$
H=-\sum_{i=1}^{N-1} J_{i} \sigma_{i} \sigma_{i+1} \quad\left(\sigma_{i}= \pm 1\right)
$$

where the $J_{i}$ are distributed according to a distribution $\rho\left(J_{i}\right)$. For simplicity, we consider only the case where the $J_{i}$ are random ferromagnetic interactions. At temperature $T$ the correlation function between two spins at distance $R$ is

$$
\left\langle\sigma_{i} \sigma_{i+R}\right\rangle=\prod_{j=i}^{i+R-1} \tanh \left(J_{j} / T\right) .
$$

From equation (2) we see that the correlation function is a product of independent random variables. For large $R$ it has the properties of the product of a large number of random variables. The probability distribution of $\left\langle\sigma_{i} \sigma_{i+R}\right\rangle$ is concentrated around its most probable value $\left\langle\sigma_{i} \sigma_{i+R}\right\rangle_{\mathrm{mp}}$

$$
\left\langle\sigma_{i} \sigma_{i+R}\right\rangle_{\mathrm{mp}} \simeq \exp [R \overline{\ln (\tanh J / T)}] \quad \text { (large } R \text { ) }
$$

whereas the average correlation function $\left.\overline{\left\langle\sigma_{i} \sigma_{i+R}\right.}\right\rangle$ is

$$
\left\langle\overline{\sigma_{i} \sigma_{i+R}}\right\rangle=\exp [R \ln \overline{(\tanh J / T)}]
$$

where $\overline{f(J)}$ means $\int \rho(J) f(J) \mathrm{d} J$.

Depending on the physical quantities one wants to study, the correlation function has to be calculated by equation (3) or equation (4). For example, if the spin $\sigma_{i}$ is fixed to be +1 and if one asks how the magnetisation $\left\langle\sigma_{i+R}\right\rangle$ decreases as a function of the distance $R$, one must use formula (3). On the other hand, if one wants to calculate the average magnetic susceptibility by summing the correlation functions, one has to use formula (4). The difference between the two can be a huge factor, as illustrated in figure 1.

The common feeling is that the average correlation function can be recovered by summing the correlation function over all the sites of the system. We shall show that this is true only up to distances $R$ of order $\ln N$. For a given sample of random interactions we define the space-averaged correlation function $g(R)$ by

$$
g(R)=N^{-1} \sum_{i=1}^{N}\left\langle\sigma_{i} \sigma_{i+R}\right\rangle .
$$

For simplicity we have assumed periodic boundary conditions $\left(\sigma_{i+N}=\sigma_{i}\right)$ here. From one sample to another, $g(R)$ fluctuates. Obviously the average of $g(R)$ is

$$
\left.\overline{g(R)}=\overline{\left\langle\sigma_{i} \sigma_{i+R}\right.}\right\rangle=a^{R}
$$

where $a=\tanh J / T$.

We can also calculate the fluctuations of $g(R)$ :

$$
\begin{aligned}
\overline{g(R)^{2}}-\overline{g(R)^{2}} & =N^{-1}\left[-2 R a^{2 R}+\frac{b^{2}+a^{2}}{b^{2}-a^{2}}\left(b^{2 R}-a^{2 R}\right)\right] \\
& \sim N^{-1} \frac{b^{2}+a^{2}}{b^{2}-a^{2}} b^{2 R}
\end{aligned}
$$

where $b^{2}=\overline{\tanh ^{2}(J / T)}$. 
(Equations (6) and (7) have been calculated with the aid of equation (2) which neglects the periodic boundary conditions. In the large $N$ limit they are correct if $R<$ $x N$ for any $x$ less than $\frac{1}{2}$. This restriction is of no importance in what follows.)

We shall call the fluctuations of $g(R)$ small if

$$
\left(\overline{g(R)^{2}}-\overline{g(R)^{2}}\right) / \overline{g(R)^{2}} \ll 1 \text {. }
$$

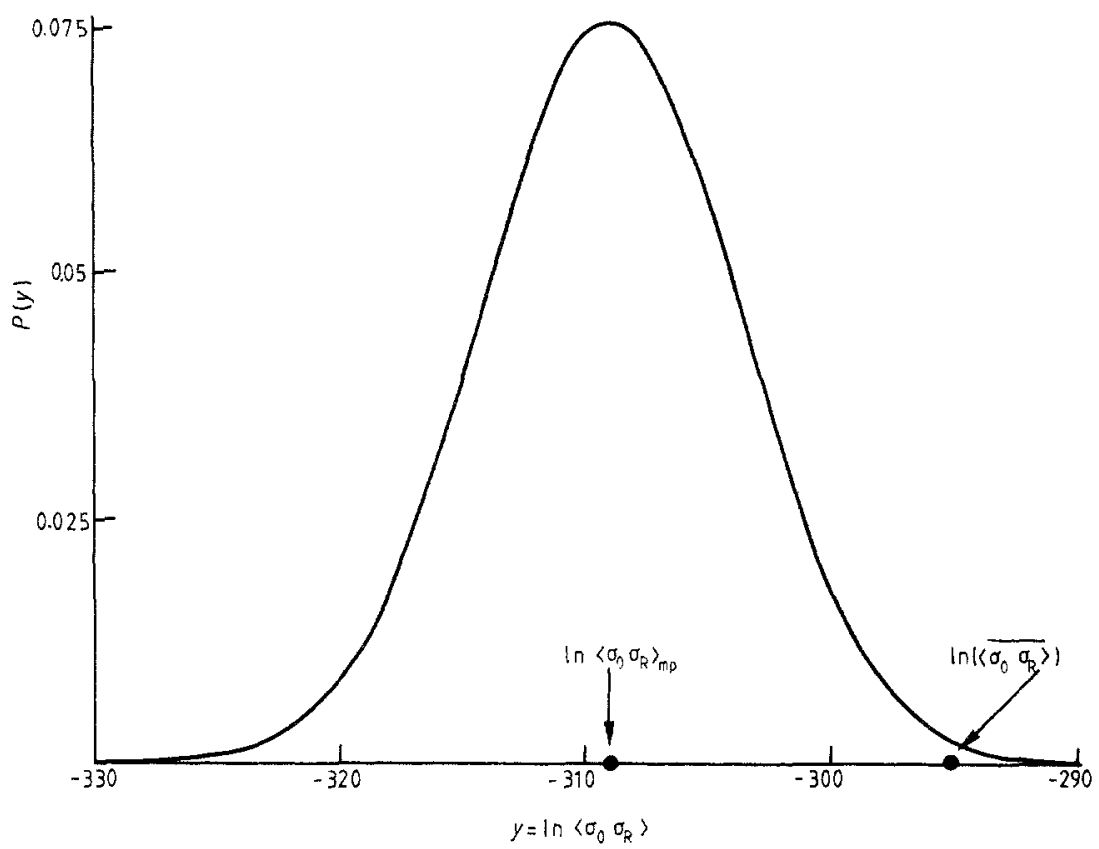

Figure 1. Probability distribution of $y=\ln \left\langle\sigma_{0} \sigma_{R}\right\rangle$ in the random Ising chain. The distance $R$ is 2000, the distribution of the random interactions is $\rho\left(J_{i}\right)=\frac{1}{2}\left[\delta\left(J_{i}-J\right)+\delta\left(J_{i}-2 J\right)\right]$ and the temperature $T$ is equal to $J$. Even with a logarithmic scale for $y$ the distribution is concentrated very far from $\left.\ln \left(\overline{\left(\sigma_{0} \sigma_{R}\right.}\right\rangle\right)$. The average $\left\langle\overline{\sigma_{0} \sigma_{R}}\right\rangle$ is about 3000 times larger than the value $\left\langle\sigma_{0} \sigma_{R}\right\rangle_{\mathrm{mp}}$ which is the most probable outcome of a measurement.

From equations (6) and (7), we find that this criterion is satisfied only if

$$
R<\ln N / \ln \left(b^{2} / a^{2}\right) .
$$

So by summing a correlation function $\left\langle\sigma_{i} \sigma_{i+R}\right\rangle$ over all positions in a given sample, one recovers the average correlation function $\overline{g(R)}$ only for distances $R$ smaller than $\ln N$. For larger distances, one obtains a result $g(R)$ which fluctuates from one sample to another and whose most probable value differs appreciably from its average.

Our second example is the cubic chain with random nearest-neighbour interactions. The Hamiltonian is

$$
H=-\sum_{i=1}^{N-1} J_{i} \boldsymbol{\sigma}_{i} \cdot \boldsymbol{\sigma}_{i+1}-J_{N} \boldsymbol{\sigma}_{N} \cdot \boldsymbol{\sigma}_{1}
$$

where the interactions are randomly distributed according to a distribution $\rho\left(J_{i}\right)$ and 
each spin is an $n$-component vector which can take the $2 n$ values

$$
\boldsymbol{\sigma}_{i}=(0,0 \ldots, \pm \sqrt{n}, 0 \ldots, 0) .
$$

The cubic model has two main interests (Balian and Toulouse 1974, Hilhorst 1977). Firstly, in the limit $n \rightarrow 0$, one recovers the self-avoiding walk problem. Secondly, for $n<1$, the model exhibits a phase transition in one dimension in the pure case (when the interactions $J_{i}$ are not random). Here, we are interested in studying how this transition is modified when the interactions are random. The partition function at temperature $T$ of the cubic chain defined by equation (10) is

$$
\begin{aligned}
Z=(2 n)^{N}\left[\prod_{i=1}^{N}\right. & \left(\frac{\cosh \left(n J_{i} / T\right)+n-1}{n}\right)+n \prod_{i=1}^{N}\left(\frac{\sinh \left(n J_{i} / T\right)}{n}\right) \\
& \left.+(n-1) \prod_{i=1}^{N}\left(\frac{\cosh \left(n J_{i} / T\right)-1}{n}\right)\right] .
\end{aligned}
$$

By looking at equation (12), one finds that there is a transition temperature $T_{\mathrm{c}}$ given by

$$
\overline{\ln \left[\cosh \left(n J / T_{c}\right)+n-1\right]}=\overline{\ln \left[\sinh \left(n J / T_{c}\right)\right]} .
$$

When $T>T_{\mathrm{c}}$, with probability 1 , the first product in equation (12) is much larger than the two others. Therefore the average free energy is given by

$$
N^{-1} \overline{\ln Z}=\ln 2+\overline{\ln [\cosh (n J / T)+n-1]} .
$$

When $T<T_{\mathrm{c}}$, with probability 1 , the partition function (12) is dominated by the second product. Therefore

$$
N^{-1} \overline{\ln Z}=\ln 2+\overline{\ln [\sinh (n J / T)]} .
$$

One notices that here, like in the pure case, the temperature $T_{\mathrm{c}}$ is finite only when $n<1$.

For this random cubic chain, the correlation function $\left\langle\boldsymbol{\sigma}_{i} \cdot \boldsymbol{\sigma}_{i+R}\right\rangle$ has also a very simple expression in the high-temperature phase

$$
\left\langle\boldsymbol{\sigma}_{i} \cdot \boldsymbol{\sigma}_{i+R}\right\rangle=n \prod_{j=i}^{i+R-1} \frac{\sinh \left(n J_{j} / T\right)}{\cosh \left(n J_{j} / T\right)+n-1} .
$$

Here again the correlation appears as a product of random numbers. For large $R$ the distribution of this correlation function is sharply peaked around its most probable value $\left\langle\boldsymbol{\sigma}_{i} \cdot \boldsymbol{\sigma}_{i+R}\right\rangle_{\mathrm{mp}}$

$$
\left\langle\boldsymbol{\sigma}_{i} \cdot \boldsymbol{\sigma}_{i+R}\right\rangle_{\mathrm{mp}} \sim \exp R\{\overline{\log [\sinh (n J / T) /(\cosh (n J / T)+n-1)]}\} .
$$

We can define a correlation length $\xi_{\mathrm{mp}}$ associated with this most probable value of the correlation function

$$
\xi_{\mathrm{mp}}^{-1}=\overline{\ln [(\cosh (n J / T)+n-1) / \sinh (n J / T)]} .
$$

One can notice that this correlation length $\xi_{\mathrm{mp}}$ diverges at the critical temperature $T_{\mathrm{c}}$ that we found in equation (13). 
If we consider the average correlation function $\left.\overline{\left\langle\boldsymbol{\sigma}_{i} \cdot \boldsymbol{\sigma}_{i+R}\right.}\right\rangle$, we find

$$
\left\langle\overline{\boldsymbol{\sigma}_{i} \cdot \boldsymbol{\sigma}_{i+R}}\right\rangle \sim \exp R\{\overline{\ln } \overline{[\sinh (n J / T) /(\cosh (n J / T)+n-1)]}\} .
$$

The correlation length $\xi_{\mathrm{a}}$ associated with this average correlation function is given by

$$
\xi_{\mathrm{a}}^{-1}=-\ln \overline{[\sinh (n J / T) /(\cosh (n J / T)+n-1)]} .
$$

This correlation length diverges at a temperature $T_{\mathrm{c}}^{\prime}$ which lies above $T_{\mathrm{c}}$. This shows that it is of crucial importance to know whether one is measuring $\xi_{\mathrm{mp}}$ or $\xi_{\mathrm{a}}$. The preceding example of the Ising model indicates that, in view of the smallness of $\ln N$, measurement of $g(R)$ leads to $\xi_{\mathrm{mp}}$ and not to $\xi_{\mathrm{a}}$, even if $R$ is only moderately large.

For large $R$ the correlation function $\left\langle\boldsymbol{\sigma}_{i} \cdot \boldsymbol{\sigma}_{i+R}\right\rangle$ is a quantity of the same nature as $Z$ as far as its properties under averaging are concerned: in many cases the quantity of physical interest is the average of the logarithm of the correlation function, and hence $\xi_{\mathrm{mp}}$, instead of $\xi_{\mathrm{a}}$.

It order to assess the behaviour of correlation functions in dimension $d>1$ we finally present a heuristic argument for the random $n$-component cubic model in the limit $n \rightarrow 0$, for arbitrary $d$.

The Hamiltonian is the one of equation (10), generalised to an arbitrary lattice, with random nearest-neighbour interactions $J_{i j}$ between sites $i$ and $j$. For $n \rightarrow 0$ the correlation function of this system is given by

$$
\left\langle\boldsymbol{\sigma}_{0} \cdot \boldsymbol{\sigma}_{R}\right\rangle=\sum_{C} \prod_{\langle i, j\rangle \in C} J_{i j}
$$

where $C$ is an arbitrary self-avoiding walk along the lattice bonds starting at 0 and ending at $R$. The fluctuations in the space-averaged correlation function $g(R)$ (defined as in (5), but with $i$ running through all $N$ sites of the $d$-dimensional lattice) will be dominated by contributions from pairs of walks occupying largely the same volume. A walk from 0 to $R$ typically (that is, for $R$ on the scale of the correlation length) occupies a volume $R^{d}$ and has a length $L=R^{1 / \nu}$. Hence it passes through a fraction $f=R^{1 / \nu-d}$ of all the bonds within its volume. If a second walk of the same length $L$ occupies the same volume, then $f L$ of its bonds will, typically, coincide with bonds of the first walk. Since coinciding bonds contribute $b^{2} \equiv \bar{J}^{2}$, and non-coinciding bonds contribute $a \equiv \bar{J}$ to the fluctuations in $g(R)$, we have the estimate

$$
\overline{g(R)^{2}}-\overline{g(R)^{2}} \sim N^{-1} C_{R}^{2} b^{2 f L} a^{2(1-f) L}
$$

where $C_{R}$ is the number of walks connecting 0 and $R$.

Upon dividing by $\overline{g(R)^{2}} \sim\left(C_{R} a^{L}\right)^{2}$ and expressing $f$ and $L$ in $R$, we see that the fluctuations in $g(R)$ are negligible only as long as

$$
R<\left(\ln N / \ln b^{2} / a^{2}\right)^{v /(2-v d)} .
$$

If we use the scaling relation $\alpha=2-\nu d$ and assume that the fluctuations of $J_{i j}$ around $\bar{J}$ are small, then equation (23) takes the form

$$
R<\left(\overline{\Delta J^{2} / J^{2}}\right)^{-v / \alpha}(\ln N)^{\nu / \alpha} \text {. }
$$

The first factor on the right-hand side is precisely the maximum correlation length that can occur, according to Harris' criterion (Harris 1974), in a system with randomness of width $\overline{\Delta J^{2}}$. For $\alpha>0$, condition (24) says that the fluctuations of $g(R)$ around its average 
become considerable as soon as $R$ exceeds the Harris correlation length by more than a factor $(\ln N)^{\nu / \alpha}$. It will usually be impracticable to increase this factor by much. Hence

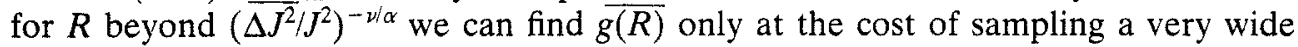
distribution of $g(R)$. This cost becomes prohibitive if the distribution develops a peak around a most probable value quite different from the average. Such is the case in the one-dimensional examples that we studied exactly, and it is plausible that the same happens for $d>1$. Therefore beyond the Harris length the only correlation length which makes sense is $\xi_{\mathrm{mp}}$, associated with the decay of $\left\langle\boldsymbol{\sigma}_{0} \cdot \boldsymbol{\sigma}_{R}\right\rangle_{\mathrm{mp}}$.

In particular, in Monte Carlo simulations where the samples are obtained randomly, there is no hope of measuring the average correlation function at long distance with any precision at all. In almost all the samples, the correlation function will take its most probable value. This makes it impossible to calculate the average susceptibility by the usual relation

$$
\bar{\chi}=(1 / T) \sum_{i j}\left(\overline{\left\langle\sigma_{i} \sigma_{j}\right\rangle}-\overline{\left\langle\sigma_{i}\right\rangle\left\langle\sigma_{j}\right\rangle}\right) .
$$

In spin glasses where the interactions are randomly ferromagnetic or antiferromagnetic, the average correlation function $\left\langle\overline{\sigma_{i} \sigma_{j}}\right\rangle$ vanishes and one usually measures the order by $\overline{\left\langle\sigma_{0} \sigma_{R}\right\rangle^{2}}$ (see for example the recent review by Morgenstern and Binder 1980). As in our two examples, $\left\langle\sigma_{0} \sigma_{R}\right\rangle^{2}$ has a distribution concentrated far away from its average when $R$ is large. This means that in Monte Carlo simulations it is impossible to measure $\left\langle\overline{\left.\sigma_{0} \sigma_{R}\right\rangle^{2}}\right.$, almost all the data being concentrated around $\left\langle\sigma_{0} \sigma_{R}\right\rangle_{\mathrm{mp}}^{2}$

\section{References}

Balian R and Toulouse G 1974 Ann. Phys., Paris 8328

Edwards S F and Anderson P W 1975 J. Phys. F: Met. Phys. 5965

Harris A B 1974 J. Phys. C: Solid State Phys. 71671

Hilhorst H 1977 J. Statist. Phys. 17413

McCoy B M and Wu T T 1968 Phys. Rev. 176631

Morgenstern I and Binder K 1980 Phys. Rev. B22 288

Sherrington D and Kirkpatrick S 1975 Phys. Rev. Lett. 351972 